\title{
A CONTINUITY PROPERTY OF HOLOMORPHIC DIFFERENTIALS UNDER QUASICONFORMAL DEFORMATIONS
}

\author{
YUKIO KUSUNOKI and MASAHIKO TANIGUCHI
}

\section{Introduction}

In this paper we shall investigate a continuity property of certain holomorphic Abelian differentials with respect to the Dirichlet norm under the quasiconformal deformation of Riemann surfaces. In the case of compact Riemann surfaces related studies have been made by L. Ahlfors, L. Bers and others. However, when we generalize such results to the class of open Riemann surfaces, we encounter many difficulties. For example, we do not generally know the existence of Teichmüller mappings, nor the existence and uniqueness of (square integrable) holomorphic differentials with prescribed periods along $A_{j}$-cycles. Hence we have to restrict our consideration to either certain classes of open Riemann surfaces over which some theorems used in the case of compact Riemann surfaces can be generalized, or certain classes of differentials on general surfaces with appropriate boundary behaviour.

In Chapter 1 we shall provide first some basic estimates for the variation under quasiconformal deformations of holomorphic differentials with fixed $A$-periods and holomorphic reproducing differentials. For such differentials we shall prove in Chapter 2 the continuity theorems with respect to the Dirichlet norm in the Teichmüller space of a given surface. In the case of compact Riemann surfaces these results are essentially due to L. Ahlfors [2], but we can show further in Chapter 3 that the continuity of holomorphic reproducing differentials still holds under the squeezing deformation about a non-dividing simple loop on a compact Riemann surface.

\section{Variation of holomorphic differentials}

1.1. For compact Riemann surfaces, L. Ahlfors [2] showed the continuity (or variation) of normal holomorphic differentials with respect to the Dirichlet norm, which played a fundamental role in his remarkable theory on Teichmüller spaces. To extend this continuity theorem to general Riemann surfaces we need 
Riemann's bilinear relation as in [2]. However, in the case of open Riemann surfaces, it does not generally hold in its classical form even if the differentials are square integrable. Thus to use a generalized bilinear relation we consider first a restricted class $O^{\prime \prime}$ of Riemann surfaces introduced by Kusunoki [10]. For the sake of convenience we recall the definition. Let $R$ be an open Riemann surface and $E=\left\{R_{n}\right\}_{n=1}^{\infty}$ a canonical exhaustion (cf. [4]) of $R$. Let $\mathscr{L}_{n}$ be the set of 1-cycles $\gamma$ in $R-\bar{R}_{1}$ such that each $\gamma$ consists of (piecewise smooth) dividing curves on $R$ and is freely homotopic to $\partial R_{\mathrm{n}}$. This means that every $\gamma \in \mathscr{L}_{n}$ consists of the same number of conrected components $\gamma_{i}\left(\gamma_{i} \cap \gamma_{j}=\emptyset, i \neq j\right)$ as $\partial R_{n}=\sum \tilde{\gamma}_{i}$ and dividing curves $\gamma_{i}$ and $\tilde{\gamma}_{i}$ are freely homotopic. We denote $\mathscr{L}_{E}=\cup_{n=1}^{\infty} \mathscr{L}_{n}$ and by $O^{\prime \prime}$ the class of Riemann surfaces whose element admits a canonical exhaustion $E$ for which the extremal length $\lambda\left(\mathscr{L}_{E}\right)$ vanishes. It is known ([10]) that $O^{\prime \prime} \varsubsetneqq O_{G}$ in general, but $O^{\prime \prime}=O_{G}$ whenever the genus is finite, and that on every Riemann surface $R$ of class $O^{\prime \prime}$ a generalized bilinear relation holds for harmonic differentials with finite Dirichlet norm.

For later use we shall extend it slightly as follows.

Proposition 1. Let $R$ be an open Riemann surface of class $O^{\prime \prime}$ and $E=\left\{R_{n}\right\}$ be a canonical exhaustion of $R$ such that $\lambda\left(\mathscr{L}_{E}\right)=0$, and let $\left\{A_{j}, B_{j}\right\}_{j=1}^{g}$ be a canonical homology basis with respect to $E$ modulo dividing cycles $\left.{ }^{1}\right)$, where $g(\leqq+\infty)$ is the genus of $R$. Then for any two square integrable closed $C^{1}$-differentials $\omega$ and $\sigma$ there is a sequence $\left\{n_{k}\right\}$ of integers for which the bilinear relation

$$
\left(\omega,{ }^{*} \sigma\right)=-\lim _{k \rightarrow \infty} \sum_{A_{j}, B_{j} \in R_{n_{k}}}\left[\int_{A_{j}} \omega \int_{B_{j}} \bar{\sigma}-\int_{B_{j}} \omega \int_{A_{j}} \bar{\sigma}\right]
$$

holds, where ${ }^{*} \sigma$ is the conjugate differential of $\sigma$. In particular, if $\omega$ and $\sigma$ have vanishing periods along all A-cycles, then we have

$$
\left(\omega,{ }^{*} \sigma\right)=-\int_{\boldsymbol{R}} \omega \wedge \bar{\sigma}=0 .
$$

Proof. By means of the orthogonal decomposition $\Gamma_{c}=\Gamma_{h}+\Gamma_{e 0}$ (cf. AhlforsSario [4]) we can write

$$
\omega=\omega_{h}+\omega_{e 0}, \text { and } \sigma=\sigma_{h}+\sigma_{e 0},
$$

where $\omega_{h}, \sigma_{h} \in \Gamma_{h}$ and $\omega_{e 0}, \sigma_{e 0} \in \Gamma_{e 0}$. Since $\Gamma_{h}, \Gamma_{e 0}$ and ${ }^{*} \Gamma_{e 0}$ are mutually orthogonal, we have

$$
\left(\omega,{ }^{*} \sigma\right)=\left(\omega_{h}, \sigma_{h}\right)
$$

Now, we know that the bilinear relation for $\omega_{h}$ and $\sigma_{h}$ holds on $R \in O^{\prime \prime}$ because (1)

1) For each $R_{\mathrm{n}}$, the subset $\left\{A_{j}, B_{j}\right\}_{j=1}^{g_{n}}, g_{n}(<\infty)$ being the genus of $R_{n}$, forms a homology basis on $R_{\mathrm{n}}\left(\bmod \partial R_{n}\right)$, for which $A_{i} \times B_{j}=\delta_{i j}, A_{i} \times A_{j}=B_{i} \times B_{j}=0$ for every $i, j=1, \ldots, g_{n}$. The intersection number $A \times B$ of two cycles $A$ and $B$ is taken here so that it has the positive signature when $B$ crosses $A$ from right to left. Note that it has the opposite signature to that in [4]. 
is valid for $\omega=\omega_{h}$ and $\sigma=\sigma_{h}$ with suitable $\left\{n_{k}\right\}$ (cf. [10]), where $\left\{n_{k}\right\}$ may depend on $\omega$ and $\sigma$. Since $\omega$ and $\sigma$ are $C^{1}$-differentials, $\omega_{e 0}$ and $\sigma_{e 0}$ belong to $\Gamma_{e 0} \cap \Gamma^{1} \subset$ $\Gamma_{e} \cap \Gamma^{1}=\Gamma_{e}^{1}$. Consequently, they are written as $\omega_{e 0}=d f$ and $\sigma_{e 0}=d g$ with $C^{2}-$ functions $f$ and $g$ on $R$. It follows that

$$
\int_{\boldsymbol{A}_{j}} \omega_{h}=\int_{\boldsymbol{A}_{j}} \omega \text { and } \int_{\boldsymbol{A}_{j}} \sigma_{h}=\int_{\boldsymbol{A}_{j}} \sigma,
$$

and analogously for $B$-periods, which proves the assertion.

1.2. Let $R_{0}$ be a marked Riemann surface of class $O^{\prime \prime}$ with a canonical homology basis $\left\{A_{j}, B_{j}\right\}_{j=1}^{g}$ modulo dividing curves as in Proposition 1 . We consider a $C^{2-}$ quasiconformal mapping $f_{R}$ of $R_{0}$ onto another Riemann surface $R$, where $C^{2}$ means the property of being continuous up to the second derivatives. Then $f_{R}$ induces on $R$ canonical homology basis modulo dividing curves, which we denote also by $\left\{A_{j}, B_{j}\right\}_{j=1}^{g}$. It is easy to see that $R$ also belongs to the class $O^{\prime \prime}$. Let $\theta_{0}$ be a holomorphic Abelian differential on $R_{0}$ with finite norm, that is, $\theta_{0} \in \Gamma_{a}\left(R_{0}\right)$. We shall now show that there exists on $R$ a unique differential $\theta_{R} \in \Gamma_{a}(R)$ having the same $A$-periods as $\theta_{0}$. Actually, we have more generally the following

Proposition 2. Let $S$ be an arbitrary open Riemann surface and $\omega$ be a closed $C^{1}$-differential square integrable on $S$. Then there exists a square integrable Abelian differential $\theta$ having the same periods with $\omega$ along all $A_{j}$-cycles where $\left\{A_{j}, B_{j}\right\}_{j=1}^{g}$ is a canonical homology basis on $S$ modulo dividing curves. In particular, if $S$ belongs to $O^{\prime \prime}$, then $\theta$ is uniquely determined.

Proof. Let $\omega=\omega_{h}+\omega_{e 0}$ be the orthogonal decomposition where $\omega_{h} \in \Gamma_{h}$ and $\omega_{e 0} \in \Gamma_{e 0}$. Since $\omega \in \Gamma^{1}, \omega_{e 0} \in \Gamma^{1}$ hence $\omega_{h}$ has the same periods as $\omega$. Set

$$
\int_{A_{j}} \omega=\int_{A_{j}} \omega_{h}=a_{j}+\sqrt{-1} \cdot b_{j}
$$

for every $j$ with real numbers $a_{j}$ and $b_{j}$. We write $\omega_{h}=\omega_{1}+\sqrt{-1} \cdot \omega_{2}$, where $\omega_{1}$ and $\omega_{2}$ are real harmonic differentials in $\Gamma_{h}$. Then $\varphi_{k}=\omega_{k}+\sqrt{-1} \cdot{ }^{*} \omega_{k}(k=1,2)$ are holomorphic and

$$
\int_{\boldsymbol{A}_{j}} \varphi_{1}=a_{j}+\sqrt{-1} \cdot c_{j}, \text { and } \int_{\boldsymbol{A}_{j}} \varphi_{2}=b_{j}+\sqrt{-1} \cdot d_{j}
$$

for every $j$ with some real $c_{j}$ and $d_{j}$. Then by Virtanen-Kusunoki's theorem (cf. [11], [12]) there exist holomorphic differentials $\theta_{1}$ and $\theta_{2}$ in $\Gamma_{a}$ such that

$$
\int_{A_{j}} \theta_{1}=a_{j} \text { and } \quad \int_{A_{j}} \theta_{2}=b_{j} \quad \text { for every } j .
$$

Hence $\theta=\theta_{1}+\sqrt{-1} \cdot \theta_{2}$ is a holomorphic differential in $\Gamma_{a}$ with the same $A_{j}$ periods $a_{j}+\sqrt{-1} \cdot b_{j}$ as $\omega$. 
Note that on $S \in O^{\prime \prime}$ every holomorphic differential with finite norm is uniquely determined by its $A$-periods, which follows from Proposition 1 .

Corollary 1. Let $R_{0}, R$ and $\theta_{0}$ be as before. There exists on $R$ a unique differential $\theta_{R} \in \Gamma_{a}(R)$ having the same A-periods with $\theta_{0}$.

Proof. First we show that the pull-back $\omega=\theta_{0} \circ f_{R}^{-1}$ belongs to $\Gamma_{c}^{1}(R)$. Writing $f_{R}^{-1}=f, J=\left|f_{z}\right|^{2}-\left|f_{\bar{z}}\right|^{2}$, and $\theta_{0}=a(w) d w$, we have

$$
\begin{gathered}
\left\|\theta_{0} \circ f\right\|_{R}^{2}=2 \int_{R}|(a \circ f)|^{2}\left[\left|f_{z}\right|^{2}+\left|f_{\bar{z}}\right|^{2}\right] d x d y \\
=2 \int_{R}|(a \circ f)|^{2}\left[\frac{\left|f_{z}\right|^{2}+\left|f_{\bar{z}}\right|^{2}}{\left|f_{z}\right|^{2}-\left|f_{\bar{z}}\right|^{2}}\right] J d x d y \leqq \frac{1+k^{2}}{1-k^{2}}\left\|\theta_{0}\right\|_{R_{0}}^{2},
\end{gathered}
$$

where $z=x+\sqrt{-1} \cdot y$ stands for the generic local parameter on $R$ and $k=\sup _{R}\left|f_{\bar{z}}\right| /\left|f_{z}\right|(<1)$ is the supremum of the modulus of the complex dilatation of $f$. Hence $\omega \in \Gamma^{1}(R)$, and it is easily checked that $\omega \in \Gamma_{c}^{1}(R)$.

Next, noting that $\int_{A_{j}} \theta_{0}=\int_{A_{j}} \omega$ for every $j$, we see from Proposition 2 that there exists a holomorphic square integrable differential $\theta_{R}$ on $R$ having the same periods as $\theta_{0}$ along $A$-cycles. q.e.d.

Remark. Let $\omega_{R} \in \Gamma^{k}(R)$ be given $(1 \leqq k \leqq \infty)$, and $f$ be a $C^{k+1}$-quasiconformal mapping from $R_{0}$ onto $R$. Then as in the proof of Corollary 1, we can show that $\omega_{R} \circ f \in \Gamma^{k}\left(R_{0}\right)$. Moreover, if $\omega_{R} \in \Gamma_{c}(R)$, then it is easily seen that $\omega_{R} \circ f \in \Gamma_{c}^{k}\left(R_{0}\right)$.

1.3. Now let $R_{0}$ again be a non-planar Riemann surface of class $O^{\prime \prime}$. Let $f$ be a $C^{2}$-quasiconformal mapping from $R_{0}$ onto $R, E=\left\{R_{n}\right\}_{n=1}^{\infty}$ be a canonical exhaustion of $R$ such that $\lambda\left(\mathscr{L}_{E}\right)=0$, and $\left\{A_{j}, B_{j}\right\}_{j=1}^{g}$ be a canonical homology basis with respect to $E$. Suppose that a differential $\theta_{R_{0}} \in \Gamma_{a}\left(R_{0}\right)$ is arbitrarily given. Then by Corollary 1 there exists a unique $\theta_{R} \in \Gamma_{a}(R)$ having the same periods as $\theta_{R_{0}}$ along all $A$-cycles, and we can show the following

Theorem 1. Let $R_{0} \in O^{\prime \prime}$ and $f$ be a $C^{2}$-quasiconformal mapping from $R_{0}$ onto $R$. Then, given $\theta_{R_{0}} \in \Gamma_{a}\left(R_{0}\right)$, there exists a unique $\theta_{R} \in \Gamma_{a}(R)$ with the same A-periods as $\theta_{R_{0}}$ and we have

$$
\left\|\theta_{R} \circ f-\theta_{R_{0}}\right\|_{R_{0}} \leqq \frac{2 k}{1-k}\left\|\theta_{R_{0}}\right\|_{R_{0}},
$$

where $k=\sup _{R_{0}}\left|f_{\bar{z}}\right| /\left|f_{z}\right|(<1)$, i.e. $K=(1+k) /(1-k)$ is the maximal dilatation of $f$.

Proof. Write $\theta_{R}=a(w) d w$ on $R$, and set $\omega=\theta_{R} \circ f-\theta_{R_{0}}$. Then, since $\omega$ has vanishing periods along all $A_{j}$-cycles, we have by Proposition 1

which implies immediately

$$
\left(\omega,{ }^{*} \omega\right)=0,
$$

$$
\left\|(a \circ f) \cdot f_{z}(z) d z-\theta_{R_{0}}\right\|_{R_{0}}^{2}-\left\|(a \circ f) \cdot f_{\bar{z}}(z) d \bar{z}\right\|_{R_{0}}^{2}=0 .
$$


As $\left|f_{\bar{z}}\right| \leqq k\left|f_{z}\right|$, we have

and hence

$$
\left\|(a \circ f) \cdot f_{z}(z) d z-\theta_{R_{0}}\right\|_{R_{0}} \leqq k\left\|(a \circ f) \cdot f_{z}(z) d z\right\|_{R_{0}},
$$

$$
\left\|(a \circ f) \cdot f_{z}(z) d z\right\|_{R_{0}} \leqq \frac{1}{1-k}\left\|\theta_{R_{0}}\right\|_{R_{0}} .
$$

Thus the assertion follows from the inequality

$$
\left\|\theta_{R} \circ f-\theta_{R_{0}}\right\|_{R_{0}} \leqq\left\|(a \circ f) \cdot f_{z}(z) d z-\theta_{R_{0}}\right\|_{R_{0}}+\left\|(a \circ f) \cdot f_{\bar{z}} d \bar{z}\right\|_{R_{0}} .
$$

1.4. Next let $R$ be an arbitrary Riemann surface, and a simple closed curve $c$ on $R$ be given. There now exists a unique differential $\theta_{c, R} \in \Gamma_{a}(R)$ which satisfies the condition

$$
\int_{c} \omega=\left(\omega, \operatorname{Re} \theta_{c, R}\right) \text { for every } \omega \in \Gamma_{h}(R) .
$$

We call $\theta_{c, R}$ the holomorphic reproducing differential for $c$ on $R$ (cf. [4], [12]). This differential has several extremal properties, and especially $\left\|\operatorname{Re} \theta_{c, R}\right\|^{2}$ $\left(=1 / 2\left\|\theta_{c, R}\right\|^{2}\right)$ is equal to the extremal length of the homology class of $c$ on $R$.

For simplicity, we write hereafter a curve corresponding to $c$ on another surface again as $c$. As for $\theta_{c, R}$ we can show the following

Theorem 2. Let $R_{0} \in O_{H D}$ and a simple closed curve $c$ be given (on $R_{0}$ ), and $f$ be a $C^{2}$-quasiconformal mapping from $R_{0}$ onto a surface $R$. Then, if we let $k=\sup _{R_{0}}\left|f_{\bar{z}}\right| /\left|f_{z}\right|$, it holds that

$$
\left\|\theta_{c, R} \circ f-\theta_{c, R_{0}}\right\|_{R_{0}} \leqq \frac{2 k}{1-k}\left\|\theta_{c, R_{0}}\right\|_{R_{0}} .
$$

Proof. Write $\omega=\theta_{c, R} \circ f-\theta_{c, R_{0}}$. Now we find $\omega \in \Gamma_{c}^{1}\left(R_{0}\right)$ as before. So $\omega$ can be decomposed in the form

$$
\omega=\omega_{1}+d g_{1}+\sqrt{-1} \cdot\left(\omega_{2}+d g_{2}\right)
$$

with real $\omega_{j} \in \Gamma_{h}\left(R_{0}\right)$ and real $d g_{j} \in \Gamma_{e 0}^{1}\left(R_{0}\right)(j=1,2)$.

Note that for every 1-cycle $d$ on $R_{0}$

$$
\operatorname{Im} \int_{d} \theta_{c, R_{0}}=c \times d=\operatorname{Im} \int_{d} \theta_{c, R} .
$$

Hence $\omega_{2} \in \Gamma_{h e}\left(R_{0}\right)$, and, further, $\omega_{2}=0$ because $R_{0} \in O_{H D}\left(\Gamma_{h e}\left(R_{0}\right)=\{0\}\right)$, and we have

$$
\begin{gathered}
\left(\omega,{ }^{*} \omega\right)=\left(\omega_{1}+d g_{1}+\sqrt{-1} \cdot d g_{2},{ }^{*} \omega_{1}+{ }^{*} d g_{1}+\sqrt{-1} * d g_{2}\right) \\
=\left(\omega_{1},{ }^{*} \omega_{1}\right)=-\int_{R_{0}} \omega_{1} \wedge \omega_{1}=0 .
\end{gathered}
$$

Thus by the same argument as in the proof of Theorem 1, we can prove the assertion. 
1.5. Finally, to obtain a similar result as Theorem 2 for an arbitrary Riemann surface, we must restrict ourselves to reproducing differentials for a suitable subclass of $\Gamma_{h}$. Namely, we consider the holomorphic $\Gamma_{h 0}$-reproducing differential $\theta_{c}\left(\Gamma_{h 0}(R)\right)$ for a given $c$ on $R$, which is characterized by the condition that $\operatorname{Re} \theta_{c}\left(\Gamma_{h 0}(R)\right) \in \Gamma_{h 0}(R)$, and

$$
\int_{c} \omega=\left(\omega, \operatorname{Re} \theta_{c}\left(\Gamma_{h 0}(R)\right)\right) \quad \text { for every } \omega \in \Gamma_{h 0}(R) .
$$

Note here that $\operatorname{Im} \theta_{c}\left(\Gamma_{h 0}(R)\right)={ }^{*} \operatorname{Re} \theta_{c}\left(\Gamma_{h 0}(R)\right)$.

Now let $R_{0}$ be an arbitrary Riemann surface, and $f$ be a $C^{2}$-quasiconformal mapping from $R_{0}$ onto a surface $R$. First we show the following

Lemma $1 . \operatorname{Re} \theta_{c}\left(\Gamma_{h 0}(R)\right) \circ f \in \Gamma_{c 0}\left(R_{0}\right) \cap \Gamma^{1}\left(R_{0}\right)$.

Proof. It is clear that $\theta_{c}\left(\Gamma_{h 0}(R)\right) \circ f \in \Gamma_{c}^{1}\left(R_{0}\right)$. Next note that the mapping $F$ defined by

$$
F(d g)=(d g) \circ f \text { for every } d g \in \Gamma_{e}^{1}(R)
$$

maps $\Gamma_{e}^{1}(R)$ onto $\Gamma_{e}^{1}\left(R_{0}\right)$.

Also we have

$$
\begin{gathered}
\left(\operatorname{Re} \theta_{c}\left(\Gamma_{h 0}(R)\right) \circ f,{ }^{*} F(d g)\right)_{R_{0}}=-\int_{R_{0}} \operatorname{Re} \theta_{c}\left(\Gamma_{h 0}(R)\right) \circ f \wedge \overline{d g \circ f} \\
=-\int_{R} \operatorname{Re} \theta_{c}\left(\Gamma_{h 0}(R)\right) \wedge \overline{d g}=\left(\operatorname{Re} \theta_{c}\left(\Gamma_{h 0}(R)\right),{ }^{*} d g\right)_{R}=0
\end{gathered}
$$

for every $d g \in \Gamma_{e}^{1}(R)$. And since $\Gamma_{e}^{1}\left(R_{0}\right)$ is dense in $\Gamma_{e}\left(R_{0}\right)$, we conclude that $\operatorname{Re} \theta_{c}\left(\Gamma_{h 0}(R)\right) \circ f \in^{*} \Gamma_{e}\left(R_{0}\right)^{\perp}=\Gamma_{c 0}\left(R_{0}\right)$.

Now we can show the following

Theorem 3. Let $R_{0}, R$ and $f$ be as above and $k=\sup _{R_{0}}\left|f_{\bar{z}}\right| /\left|f_{z}\right|$. Then it holds that

$$
\left\|\theta_{c}\left(\Gamma_{h 0}(R)\right) \circ f-\theta_{c}\left(\Gamma_{h 0}\left(R_{0}\right)\right)\right\|_{R_{0}} \leqq \frac{2 k}{1-k}\left\|\theta_{c}\left(\Gamma_{h 0}\left(R_{0}\right)\right)\right\|_{R_{0}} .
$$

Proof. Write $\omega=\theta_{c}\left(\Gamma_{h 0}(R)\right) \circ f-\theta_{c}\left(\Gamma_{h 0}\left(R_{0}\right)\right)$. Then by Lemma $1 \operatorname{Re} \omega \in \Gamma_{c 0}\left(R_{0}\right) \cap$ $\Gamma^{1}\left(R_{0}\right)$, and by the orthogonal decomposition $\Gamma_{c 0}\left(R_{0}\right)=\Gamma_{h 0}\left(R_{0}\right)+\Gamma_{e 0}\left(R_{0}\right)$ we can decompose $\omega$ in the form

$$
\omega=\omega_{1}+d g_{1}+\sqrt{-1} \cdot\left(\omega_{2}+d g_{2}\right),
$$

where $\omega_{j}$ and $d g_{j}$ are real, $\omega_{1} \in \Gamma_{h 0}\left(R_{0}\right), \omega_{2} \in \Gamma_{h}\left(R_{0}\right)$ and $d g_{j} \in \Gamma_{e 0}\left(R_{0}\right) \cap \Gamma^{1}\left(R_{0}\right) \subset$ $\Gamma_{e}^{1}\left(R_{0}\right)(j=1,2)$. Moreover, we know that $\omega_{2} \in \Gamma_{h e}\left(R_{0}\right)$, for it holds as before that

$$
\int_{d} \omega_{2}=\operatorname{Im} \int_{d} \theta_{c}\left(\Gamma_{h 0}(R)\right)-\operatorname{Im} \int_{d} \theta_{c}\left(\Gamma_{h 0}\left(R_{0}\right)\right)=0
$$


for every 1-cycle $d$. So noting that $\left(\omega_{1},{ }^{*} \omega_{2}\right)=0$ because $\omega_{1} \in \Gamma_{h 0}\left(R_{0}\right)$ and ${ }^{*} \omega_{2} \in^{*} \Gamma_{h e}\left(R_{0}\right)=\Gamma_{h 0}\left(R_{0}\right)^{\perp} \cap \Gamma_{h}\left(R_{0}\right)$, we have

$$
\begin{gathered}
\left(\omega,{ }^{*} \omega\right)=\left(\omega_{1}+d g_{1}+\sqrt{-1} \cdot\left(\omega_{2}+d g_{2}\right),{ }^{*} \omega_{1}+{ }^{*} d g_{1}+\sqrt{-1} \cdot\left({ }^{*} \omega_{2}+{ }^{*} d g_{2}\right)\right) \\
=-2 \sqrt{-1} \cdot\left(\omega_{1},{ }^{*} \omega_{2}\right)=0 .
\end{gathered}
$$

Thus by the same argument as in the proof of Theorem 1, we have the assertion.

Corollary 2. Let $R, R_{0}$ and f be as in Theorem 3 , and $\theta_{c}\left(\Gamma_{\text {hse }}\left(R_{0}\right)\right)$ and $\theta_{c}\left(\Gamma_{\text {hse }}(R)\right)$

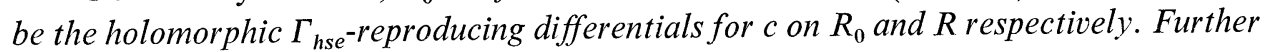
suppose that $R_{0} \in O_{K D}$. Then it holds that

$$
\left\|\theta_{c}\left(\Gamma_{h s e}(R)\right) \circ f-\theta_{c}\left(\Gamma_{h s e}\left(R_{0}\right)\right)\right\|_{R_{0}} \leqq \frac{2 k}{1-k}\left\|\theta_{c}\left(\Gamma_{h s e}\left(R_{0}\right)\right)\right\|_{R_{0}} .
$$

This follows from Theorem 3 , since $R_{0} \in O_{K D}$ if and only if $\Gamma_{h s e}\left(R_{0}\right)=\Gamma_{h 0}\left(R_{0}\right)$.

\section{Continuity theorem on the Teichmüller spaces}

2.1. Let $R^{*}$ be a fixed Riemann surface with the hyperbolic universal covering surface, and consider all pairs $(R, f)$, where $R$ is a Riemann surface and $f$ is a quasiconformal mapping from $R^{*}$ onto $R$. We say that $\left(R_{1}, f_{1}\right)$ and $\left(R_{2}, f_{2}\right)$ are equivalent if $f_{2} \circ f_{1}^{-1}$ is homotopic to a conformal mapping from $R_{1}$ onto $R_{2}$. The equivalence classes are, by definition, the points of the Teichmüller space $T\left(R^{*}\right)$ with the base point $\bar{R}^{*}=\left(R^{*}, \mathrm{id}\right)$, which are called marked Riemann surfaces and denoted simply by $\bar{R}$ etc. The space $T\left(R^{*}\right)$ has the natural Teichmüller metric. (See for example [3].) Now let $G^{*}$ be a Fuchsian group representing $R^{*}$, then $T\left(R^{*}\right)$ can be canonically identified with the reduced Teichmüller space $T^{\#}\left(G^{*}\right)$, which coincides with the Teichmüller space $T\left(G^{*}\right)$ if and only if $G^{*}$ is of the first kind. Also note that $\operatorname{dim} T\left(R^{*}\right)$ is finite if and only if $G^{*}$ is finitely generated (cf. [8]) and that if $R^{*} \in O_{H D}$ then $G^{*}$ is of the first kind ([13]).

In the case of general open Riemann surfaces, the existence of the so-called Teichmüller mappings is not known. Actually, for given $\bar{R}_{1}$ and $\bar{R}_{2}$ in $T\left(R^{*}\right)$ there exists an extremal quasiconformal mapping $f$ from $\bar{R}_{1}$ to $\bar{R}_{2}$ (which preserves the markings), but such an $f$ is not unique in general, and even if it were, it would not be known whether $f$ has such regularity as Teichmüller mappings. Hence we want to begin by showing the existence of a smooth quasiconformal mapping between points on $T\left(R^{*}\right)$ sufficiently near each other.

2.2. Let $\bar{R}_{0} \in T\left(R^{*}\right)$ be fixed in the sequel, and $\lambda_{\bar{R}_{0}}$ be the Poincaré metric on $R_{0}$. We say that a Beltrami differential $\mu$ on $R_{0}$ is canonical if the quadratic differential $\bar{\mu} \cdot \lambda_{\bar{R}_{0}}^{2}$ is holomorphic on $R_{0}$, and that a quasiconformal mapping $f$ from $R_{0}$ onto another surface is canonical if the complex dilatation $\mu(f)$ of $f$ is a canonical Beltrami differential. In particular, a canonical Beltrami differential has a coefficient 
of $C^{\omega}$-class and hence a canonical quasiconformal mapping is of $C^{\omega}$-class (cf. for example [6]). Now let $d($,$) be the Teichmüller distance on T\left(R^{*}\right)$, and set

$$
k(\bar{R})=\frac{\exp \left[d\left(\bar{R}_{0}, \bar{R}\right)\right]-1}{\exp \left[d\left(\bar{R}_{0}, \bar{R}\right)\right]+1}
$$

for every $\bar{R} \in T\left(R^{*}\right)$. Then by definition $k(\bar{R})$ is equal to the $L^{\infty}$-norm of the complex dilatation of any extremal quasiconformal mapping from $\bar{R}_{0}$ to $\bar{R}$.

We do not know whether a canonical quasiconformal mapping from $\bar{R}_{0}$ to $\bar{R}$ exists for every $\bar{R} \in T\left(R^{*}\right)$, but we can show the following

Lemma 2. Suppose that $k(\bar{R})<1 / 3$ (i.e. $\left.d\left(\bar{R}_{0}, \bar{R}\right)<\log 2\right)$. Then there exists a canonical quasiconformal mapping from $\bar{R}_{0}$ to $\bar{R}$ whose maximal dilatation is not greater than $(1+3 k(\bar{R})) /(1-3 k(\bar{R}))$.

Proof. Let $f$ be an extremal quasiconformal mapping from $\bar{R}_{0}$ to $\bar{R}$, and $\mu$ be the complex dilatation of $f$. Recall that ess $\sup _{R_{0}}|\mu|=k(\bar{R})$. Let $G_{0}$ be a Fuchsian group acting on the unit disk $U$ such that $R_{0}=U / G_{0}$. Then $\mu$ can be lifted to a Beltrami coefficient for $G_{0}$ on $U$, which is also denoted by $\mu$. Now there exists a unique quasi-conformal automorphism $F$ of the extended $z$-plane $\bar{C}$ fixing $1, \sqrt{-1}$ and $-\sqrt{-1}$ whose complex dilatation is $\mu$ on $U$ and zero on $\bar{C}-U$. Next let $\varphi_{F}$ be the Schwarzian derivative of $F$ considered as a schlicht function on $\bar{C}-\bar{U}$. Then Kühnau-Lehto's theorem ([9], [15]) states that

$$
\left(1-|z|^{2}\right)^{2}\left|\varphi_{F}(z)\right| \leqq 6 \cdot k(\bar{R})(<2) \text { on } \bar{C}-\bar{U} .
$$

This in turn implies (cf. [3], [5]) that there exists a unique quasiconformal automorphism $g$ on $\overline{\boldsymbol{C}}$ such that

(i) the complex dilatation of $g$ is equal to

$$
\left\{\begin{array}{c}
-\frac{1}{2}\left(1-|z|^{2}\right)^{2} \varphi_{F}\left(\frac{1}{\bar{z}}\right)\left(\frac{1}{\bar{z}}\right)^{4} \text { on } U, \text { and } \\
0 \text { on } \bar{C}-U, \text { and }
\end{array}\right.
$$

(ii) $g(z) \equiv F(z)$ on $\bar{C}-U$.

Now by (i) it is seen that the complex dilatation of $g$ is a Beltrami coefficient for $G_{0}$, and (ii) implies that $F \circ G_{0} \circ F^{-1}=g \circ G_{0} \circ g^{-1}$ on $\bar{C}$. Thus $g$ can be projected to a quasiconformal mapping from $R_{0}$ onto $R\left(=F(U) / F \circ G_{0} \circ F^{-1}\right)$, which is canonical by (i). And noting that

we have the assertion.

$$
\begin{aligned}
& \sup _{U}\left|-\frac{1}{2}\left(1-|z|^{2}\right)^{2} \varphi_{F}\left(\frac{1}{\bar{z}}\right)\left(\frac{1}{\bar{z}}\right)^{4}\right| \\
= & \sup _{\bar{C}-U}\left|-\frac{1}{2}\left(1-|z|^{2}\right)^{2} \varphi_{F}(z)\right| \leqq 3 \cdot k(\bar{R}),
\end{aligned}
$$


2.3. By Lemma 2 above one can restate Theorem 1 as follows.

Theorem $1^{\prime}$. Let $R^{*} \in O^{\prime \prime}$, and a point $\bar{R}_{0} \in T\left(R^{*}\right)$ and $\theta_{\bar{R}_{0}} \in \Gamma_{a}\left(R_{0}\right)$ be given. Suppose that $d\left(\bar{R}_{0}, \bar{R}\right)<\log 2$, and let $\theta_{\bar{R}}$ be the differential in $\Gamma_{a}(R)$ having the same A-periods with $\theta_{\bar{R}_{0}}$. Then there exists a canonical quasiconformal mapping $f_{\bar{R}}$ from $\bar{R}_{0}$ to $\bar{R}$ such that

$$
\left\|\theta_{\bar{R}} \circ f_{\bar{R}}-\theta_{\bar{R}_{0}}\right\|_{R_{0}} \leqq \frac{6 \cdot k(\bar{R})}{1-3 \cdot k(\bar{R})}\left\|\theta_{\bar{R}_{0}}\right\|_{R_{0}} .
$$

Corollary 3. Using the same notation as in Theorem 1', we have

$$
\lim _{\bar{R} \rightarrow \bar{R}_{0}}\left\|\theta_{\bar{R}}\right\|_{R}=\left\|\theta_{\bar{R}_{0}}\right\|_{R_{0}}, \quad \text { and }
$$

$$
\lim _{\bar{R} \rightarrow \bar{R}_{0}} \int_{d} \theta_{\bar{R}}=\int_{d} \theta_{\bar{R}_{0}} \text { for every } 1 \text {-cycle } d \text {. }
$$

Proof. For $\bar{R}$ sufficiently near $\bar{R}_{0}$ there is a canonical quasiconformal mapping $f_{\bar{R}}$ of $R_{0}$ onto $R$. Let $\theta_{\bar{R}}=a_{\bar{R}}(w) d w$ with $w=f_{\bar{R}}(z)$. Then

$$
\begin{gathered}
\left\|\theta_{\bar{R}}\right\|_{R}=\left[2 \int_{R_{0}}\left|a_{\bar{R}} \circ f_{\bar{R}}\right|^{2}\left(\left|\left(f_{\bar{R}}\right)\right|_{z}^{2}-\left|\left(f_{\bar{R}}\right)_{\bar{z}}^{2}\right|\right) d x d y\right]^{1 / 2} \\
\leqq\left\|\theta_{\bar{R}} \circ f_{\bar{R}}\right\|_{R_{0}} \leqq\left\|\theta_{\bar{R}} \circ f_{\bar{R}}-\theta_{\bar{R}_{0}}\right\|_{R_{0}}+\left\|\theta_{\bar{R}_{0}}\right\|_{R_{0}} .
\end{gathered}
$$

Hence by Theorem $1^{\prime}$ we see that

$$
\limsup _{\bar{R} \rightarrow R_{0}}\left\|\theta_{\bar{R}}\right\|_{R} \leqq\left\|\theta_{\bar{R}_{0}}\right\|_{R_{0}} .
$$

On the other hand, writing $k=k(\bar{R})$, we have

$$
\begin{gathered}
\left\|\theta_{\bar{R}_{0}}\right\|_{R_{0}} \leqq\left\|\theta_{\bar{R}} \circ f_{\bar{R}}-\theta_{\bar{R}_{0}}\right\|_{R_{0}}+\left\|\theta_{\bar{R}} \circ f_{\bar{R}}\right\|_{R_{0}} \\
\leqq\left\|\theta_{\bar{R}} \circ f_{\bar{R}}-\theta_{\bar{R}_{0}}\right\|_{R_{0}}+\left[\frac{1+k^{2}}{1-k^{2}}\right]^{1 / 2}\left\|\theta_{\bar{R}}\right\|_{R},
\end{gathered}
$$

and consequently the inequality

$$
\liminf _{\bar{R} \rightarrow \bar{R}_{0}}\left\|\theta_{\bar{R}}\right\|_{R} \geqq\left\|\theta_{\bar{R}_{0}}\right\|_{R_{0}} .
$$

It is well-known that for a fixed 1-cycle $d$, the linear functional $L(\omega)=\int_{d} \omega$ is bounded on $\Gamma_{c}^{1}\left(R_{0}\right)$, that is, there is a constant $C_{d}$ such that

$$
\left|\int_{d} \omega\right| \leqq C_{d}\|\omega\|_{R_{0}} \text { for every } \omega \in \Gamma_{c}^{1}\left(R_{0}\right) .
$$

Noting that $\theta_{\bar{R}} \circ f_{\bar{R}} \in \Gamma_{c}^{1}\left(R_{0}\right)$ we have therefore

$$
\left|\int_{d} \theta_{\bar{R}}-\int_{d} \theta_{\bar{R}_{0}}\right| \leqq C_{d}\left\|\theta_{\bar{R}} \circ f_{\bar{R}}-\theta_{\bar{R}_{0}}\right\|_{R_{0}} .
$$

Thus the assertion 2) follows from Theorem $1^{\prime}$, 
Remark. The equation 2) in Corollary 3 implies that when $R \in O^{\prime \prime}$ and a canonical homology basis $\left\{A_{j}, B_{j}\right\}$ on $R$ is suitably chosen, then the period matrix with respect to this basis varies continuously on $T(R)$ (equipped with the Teichmüller topology).

2.4. Similarly we have the following results from Theorems 2 and 3 respectively.

Theorem $2^{\prime}$. Let $R^{*} \in O_{H D}$ and a simple closed curve $c$ be given. Suppose that $d\left(\bar{R}_{0}, \bar{R}\right)<\log 2$. Then there exists a canonical quasiconformal mapping $f_{\bar{R}}$ from $\bar{R}_{0}$ to $\bar{R}$ for which

$$
\left\|\theta_{c, \bar{R}} \circ f_{\bar{R}}-\theta_{c, \bar{R}_{0}}\right\|_{R_{0}} \leqq \frac{6 \cdot k(\bar{R})}{1-3 \cdot k(\bar{R})}\left\|\theta_{c, \bar{R}_{0}}\right\|_{R_{0}} .
$$

Corollary 4. Using the same notation as in Theorem 2', we have

$$
\lim _{\bar{R} \rightarrow \bar{R}_{0}}\left\|\theta_{c, \bar{R}}\right\|_{R}=\left\|\theta_{c, \bar{R}_{0}}\right\|_{R_{0}} \text {, and }
$$

$$
\lim _{\bar{R} \rightarrow \bar{R}_{0}} \int_{d} \theta_{c, \bar{R}}=\int_{d} \theta_{c, \bar{R}_{0}} \text { for every 1-cycle } d .
$$

Theorem $3^{\prime}$. Let $R^{*}$ and a simple closed curve $c$ be arbitrarily given. Suppose that $d\left(\bar{R}_{0}, \bar{R}\right)<\log 2$. Then there exists a canonical quasiconformal mapping $f_{\bar{R}}$ from $\bar{R}_{0}$ to $\bar{R}$ for which

$$
\left\|\theta_{c}\left(\Gamma_{h 0}(R)\right) \circ f_{\bar{R}}-\theta_{c}\left(\Gamma_{h 0}\left(R_{0}\right)\right)\right\|_{R_{0}} \leqq \frac{6 \cdot k(\bar{R})}{1-3 \cdot k(\bar{R})}\left\|\theta_{c}\left(\Gamma_{h 0}(R)\right)\right\|_{R_{0}} .
$$

Corollary 5. Using the same notation as in Theorem 3', we have

$$
\begin{gathered}
\lim _{R \rightarrow R_{0}}\left\|\theta_{c}\left(\Gamma_{h 0}(R)\right)\right\|_{R}=\left\|\theta_{c}\left(\Gamma_{h 0}\left(R_{0}\right)\right)\right\|_{R_{0}}, \quad \text { and } \\
\lim _{R \rightarrow R_{0}} \int_{d} \theta_{c}\left(\Gamma_{h 0}(R)\right)=\int_{d} \theta_{c}\left(\Gamma_{h 0}\left(R_{0}\right)\right) \quad \text { for every 1-cycle } d .
\end{gathered}
$$

2.5. Here we shall consider quasiconformal mappings not necessarily of class $C^{2}$. Let $\bar{R}_{n} \in T\left(R^{*}\right)$ converge to $\bar{R}_{0} \in T\left(R^{*}\right)$, where $R^{*}$ is arbitrarily given, and let $f_{n}$ be a quasiconformal mapping of $\bar{R}_{0}$ to $\bar{R}_{n}$. We call a sequence $\left\{f_{n}\right\}_{n=1}^{\infty}$ admissible if the maximal dilatation of $f_{n}$ converges to 1 . Then we can show the following

Proposition 3. If we let $\theta_{\bar{R}_{n}}$ be a holomorphic Abelian differential on $R_{n}$ with finite Dirichlet norm for every $n$, then the following two conditions are equivalent;

1) For some admissible sequence $\left\{f_{n}\right\}_{n=1}^{\infty}$ we have

$$
\lim _{n \rightarrow \infty}\left\|\theta_{\bar{R}_{n}} \circ f_{n}-\theta_{\bar{R}_{0}}\right\|_{R_{0}}=0 .
$$

2) For any admissible sequence $\left\{f_{n}\right\}_{n=1}^{\infty}$, the above (3) holds. 
Proof. It is clear that 2) implies 1). Now suppose that for an admissible sequence $\left\{f_{n}\right\}_{n=1}^{\infty}$ the condition (3) holds, and let any admissible $\left\{g_{n}\right\}_{n=1}^{\infty}$ be given. First note that

$$
\begin{gathered}
\left\|\theta_{\bar{R}_{n}} \circ g_{n}-\theta_{\bar{R}_{0}}\right\|_{R_{0}}^{2} \\
\leqq 2 \frac{1+k_{n}^{2}}{1-k_{n}^{2}}\left\|\theta_{\bar{R} n} \circ g_{n} \circ\left(g_{n}^{-1} \circ f_{n}\right)-\theta_{\bar{R}_{0}} \circ\left(g_{n}^{-1} \circ f_{n}\right)\right\|_{R_{0}}^{2},
\end{gathered}
$$

where $k_{n}=\operatorname{ess}_{R_{0}} \sup \left|\left(g_{n}^{-1} \circ f_{n}\right)_{\bar{z}}\right| /\left|\left(g_{n}^{-1} \circ f_{n}\right)_{z}\right|$. In fact, for any square integrable differential $\theta=a(w) d w+b(w) d \bar{w}$, and any quasi-conformal mapping $w=F(z)$ of $R_{0}$ onto itself, it holds that

$$
\begin{aligned}
& \|\theta \circ f\|_{R_{0}}^{2}=\int_{R_{0}} 2\left(\left|a \circ F(z) \cdot F_{z}(z)+b \circ F(z) \cdot \bar{F}_{z}(z)\right|^{2}\right. \\
& \left.+\left|a \circ F(z) \cdot F_{\bar{z}}(z)+b \circ F(z) \cdot \bar{F}_{\bar{z}}(z)\right|^{2}\right) d x d y \\
& \leqq \\
& \quad 4 \int_{R_{0}}\left(|a \circ F|^{2}+|b \circ F|^{2}\right)\left(\left|F_{z}\right|^{2}+\left|F_{\bar{z}}\right|^{2}\right) d x d y \\
& \leqq 4 \frac{1+k^{2}}{1-k^{2}} \int_{R_{0}}\left(|a \circ F|^{2}+|b \circ F|^{2}\right) \cdot J d x d y \\
& =4 \frac{1+k^{2}}{1-k^{2}} \int_{R_{0}}\left(|a(w)|^{2}+|b(w)|^{2}\right) d u d v=2 \frac{1+k^{2}}{1-k^{2}}\|\theta\|_{R_{0}}^{2},
\end{aligned}
$$

where $k=\operatorname{ess}_{R_{0}} \sup \left|F_{\bar{z}}\right| /\left|F_{z}\right|, \quad J=\left|F_{z}\right|^{2}-\left|F_{\bar{z}}\right|^{2} \quad$ with generalized $L^{2}$-derivatives $F_{z}$ and $F_{\bar{z}}$. So, putting $\theta=\theta_{\bar{R}_{n}} \circ g_{n} \circ\left(g_{n}^{-1} \circ f_{n}\right)-\theta_{\bar{R}_{0}} \circ\left(g_{n}^{-1} \circ f_{n}\right)$, and $F=\left(g_{n}^{-1} \circ f_{n}\right)^{-1}$, we have (4).

Next

$$
\begin{gathered}
\left\|\theta_{\bar{R}_{n}} \circ g_{n} \circ\left(g_{n}^{-1} \circ f_{n}\right)-\theta_{\bar{R}_{0}} \circ\left(g_{n}^{-1} \circ f_{n}\right)\right\|_{R_{0}} \\
\leqq\left\|\theta_{\bar{R}_{n}} \circ f_{n}-\theta_{\bar{R}_{0}}\right\|_{R_{0}}+\left\|\theta_{\bar{R}_{0}}-\theta_{\bar{R}_{0}} \circ\left(g_{n}^{-1} \circ f_{n}\right)\right\|_{R_{0}},
\end{gathered}
$$

and the first term of the right hand side converges to 0 by assumption. To complete the proof it therefore suffices to show that

$$
\lim _{n \rightarrow \infty}\left\|\theta_{\bar{R}_{0}}-\theta_{\bar{R}_{0}} \circ\left(g_{n}^{-1} \circ f_{n}\right)\right\|_{R_{0}}=0 .
$$

For this purpose, let $G_{0}$ be a Fuchsian group acting on $U=\{|z|<1\}$ such that $R_{0}=U / G_{0}$, let $a(z) d z$ with holomorphic $a(z)$ be the lift of $\theta_{\bar{R}_{0}}$ and $F_{n}(z)$ be the lift of $\left(g_{n}^{-1} \circ f_{n}\right)$ such that $F_{n} \circ g=g \circ F_{n}$ for every $g \in G_{0}$. Note that $g_{n}$ and $f_{n}$ are homotopic. Fix a normal fundamental region $D$ for $G_{0}$ and any positive $\varepsilon$. Then there exists an $r_{0}<1$ such that $\|a(z)\|_{2, D-D_{r_{0}}}<\varepsilon$, where $D_{r}=D \cap\{|z|<r\}$ and $\|f\|_{2, E}^{2}=$ $2 \int_{E}|f|^{2} d x d y$ for any subset $E$ in $U$. Since $\bar{D}_{r}$ is compact in $U$ for any $r<1,|a(z)|$ is bounded on $D_{r}$. Since $\left\{f_{n}\right\}$ and $\left\{g_{n}\right\}$ are admissible and homotopic, $F_{n}(z)$ converges uniformly to the identity mapping on $D_{r}$, and

$$
\lim _{n \rightarrow \infty}\left\|\left(F_{n}\right)_{z}-1\right\|_{2, D_{r}}=\lim _{n \rightarrow \infty}\left\|\left(F_{n}\right)_{\bar{z}}\right\|_{2, D r}=0
$$


(Cf. [14] IV Theorem 5.2 and V Theorem 5.3.) Hence we conclude that

$$
\begin{aligned}
& \quad \limsup _{n \rightarrow \infty}\left\|\theta_{\bar{R}_{0}}-\theta_{\bar{R}_{0}} \circ\left(g_{n}^{-1} \circ f_{n}\right)\right\|_{R_{0}} \\
& \leqq \\
& +\limsup _{n \rightarrow \infty}\left[\left\|a \circ F_{n} \cdot\left[\left(F_{n}\right)_{z}-1\right]\right\|_{2, D_{r}}+\left\|a \circ F_{n} \cdot\left(F_{n}\right)_{\bar{z}}\right\|_{2, D_{r}}\right. \\
& +\left\|a-a \circ F_{n}\right\|_{2, D_{r}}+\|a\|_{2, D-D_{r}} \\
& \left.+\left(\int_{D-D_{r}} 2\left|a \circ F_{n}\right|^{2}\left(\left|\left(F_{n}\right)_{z}\right|^{2}+\left|\left(F_{n}\right)_{\bar{z}}\right|^{2}\right) d x d y\right)^{1 / 2}\right] \\
& \leqq\|a(z)\|_{2, D-D_{r}}+\limsup _{n \rightarrow \infty}\left(\frac{1+k_{n}^{2}}{1-k_{n}^{2}}\right)^{1 / 2} \cdot\|a(z)\|_{2, F_{n}\left(D-D_{r}\right)} \\
& \leqq 2 \varepsilon,
\end{aligned}
$$

where $r_{0}<r<1$. Since $\varepsilon$ is arbitrary, we have (5).

Thus using Proposition 3 we obtain from Theorem $1^{\prime}, 2^{\prime}$ and $3^{\prime}$ the following theorems.

Theorem 4. Let $R^{*} \in O^{\prime \prime}, \bar{R}_{n} \in T\left(R^{*}\right)$ converge to $\bar{R}_{0} \in T\left(R^{*}\right)$, and $\theta_{\bar{R}_{n}}$ be as in Theorem 1'. Then for every admissible sequence $\left\{f_{n}\right\}_{n=1}^{\infty}$

$$
\lim _{n \rightarrow \infty}\left\|\theta_{\bar{R}_{n}} \circ f_{n}-\theta_{\bar{R}_{0}}\right\|_{R_{0}}=0 .
$$

Theorem 5. Let $R^{*} \in O_{H D}$, and $\bar{R}_{n} \in T\left(R^{*}\right)$ converge to $\bar{R}_{0} \in T\left(R^{*}\right)$. Then for every admissible sequence $\left\{f_{n}\right\}_{n=1}^{\infty}$

$$
\lim _{n \rightarrow \infty}\left\|\theta_{c, \bar{R}_{n}} \circ f_{n}-\theta_{c, \bar{R}_{0}}\right\|_{R_{0}}=0 .
$$

Theorem 6. Let $R^{*}$ be arbitrary and $\bar{R}_{n} \in T\left(R^{*}\right)$ converge to $\bar{R}_{0} \in T\left(R^{*}\right)$. Then for every admissible sequence $\left\{f_{n}\right\}_{n=1}^{\infty}$

$$
\lim _{n \rightarrow \infty}\left\|\theta_{c}\left(\Gamma_{h 0}\left(R_{n}\right)\right) \circ f_{n}-\theta_{c}\left(\Gamma_{h 0}\left(R_{0}\right)\right)\right\|_{R_{0}}=0 .
$$

2.6. Finally we note that Theorem 5 can be extended to arbitrary Riemann surfaces. That is, we can prove the following

Theorem $5^{\prime}$. Let $R^{*}$ be an arbitrary Riemann surface, a simple closed curve $c$ be fixed, and $\bar{R}_{n} \in T\left(R^{*}\right)$ converge to $\bar{R}_{0} \in T\left(R^{*}\right)$. Then for every admissible sequence $\left\{f_{n}\right\}_{n=1}^{\infty}$

$$
\lim _{n \rightarrow \infty}\left\|\theta_{c, R_{n}} \circ f_{n}-\theta_{c, R_{0}}\right\|_{R_{0}}=0 .
$$

Proof. Suppose first that every $f_{n}$ is canonical, and let $\sigma_{c, \bar{R}_{n}}=\operatorname{Re} \theta_{c, \bar{R}_{n}}$ for every $n$. Then one can see that $\left\|\sigma_{c, R_{n}}\right\|_{R_{n}}$ converges to $\left\|\sigma_{c, \bar{R}_{0}}\right\|_{R_{0}}$, for $\left\|\sigma_{c, \bar{R}}\right\|_{R}^{2}$ is equal to the extremal length of the homology class of $c$ on $R$. Hence it holds that

$$
K_{n}^{-1}\left\|\sigma_{c, R_{0}}\right\|_{R_{0}}^{2} \leqq\left\|\sigma_{c, R_{n}}\right\|_{R_{0}}^{2} \leqq K_{n}\left\|\sigma_{c, R_{0}}\right\|_{R_{0}}^{2},
$$


where $K_{n}$ is the maximal dilatation of $f_{n}$. Note also that

$$
\begin{gathered}
\left\|\sigma_{c, \bar{R}_{n}} \circ f_{n}-\sigma_{c, \bar{R}_{0}}\right\|_{R_{0}}^{2} \\
=\left\|\sigma_{c, \bar{K}_{n}} \circ f_{n}\right\|_{R_{0}}^{2}-2 \int_{c} \sigma_{c, \bar{R}_{i}}+\left\|\sigma_{c, \bar{R}_{0}}\right\|_{R_{0}}^{2} \\
=\left\|\sigma_{c, \bar{R}_{n}} \circ f_{n}\right\|_{R_{0}}^{2}-2\left\|\sigma_{c, \bar{R}_{n}}\right\|_{R_{n}}^{2}+\left\|\sigma_{c, \bar{R}_{0}}\right\|_{R_{0}}^{2} .
\end{gathered}
$$

Now set $\theta_{c, \bar{R}_{n}}=a_{n}\left(z_{n}\right) d z_{n}$, (hence $\left.2 \sigma_{c, \bar{R}_{n}}=a_{n}\left(z_{n}\right) d z_{n}+\bar{a}_{n}\left(z_{n}\right) d \bar{z}_{n}\right)$, on $R_{n}$ with $z_{n}=f_{n}(z) . \quad$ Then $\quad \sigma_{c, \bar{R}_{n}} \circ f_{n}=(1 / 2)\left[\left(a_{n} \circ f_{n} \cdot\left(f_{n}\right)_{z}+\overline{a_{n} \circ f_{n} \cdot\left(f_{n}\right)_{\bar{z}}}\right) d z+\left(\overline{a_{n} \circ f_{n} \cdot\left(f_{n}\right)_{z}}+\right.\right.$ $\left.\left.a_{n} \circ f_{n} \cdot\left(f_{n}\right)_{\bar{z}}\right) d \bar{z}\right]$, and thus we have

$$
\begin{gathered}
\left\|\sigma_{c, \bar{R}_{n}} \circ f_{n}\right\|_{R_{0}}^{2} \\
=\frac{1}{2} \|\left[\left(a_{n} \circ f_{n} \cdot\left(f_{n}\right)_{z}+a_{n} \circ f_{n} \cdot\left(f_{n}\right)_{\bar{z}}\right] d z \|_{R_{0}}^{2}\right. \\
\leqq \frac{1}{2} \int_{R_{n}}\left|a_{n} \circ f_{n}\right|^{2}\left(\left|\left(f_{n}\right)_{z}\right|+\left|\left(f_{n}\right)_{\bar{z}}\right|\right)^{2} 2 d x d y \\
\leqq \frac{1}{2} K_{n}\left\|\theta_{c, \bar{R}_{n}}\right\|_{R_{n}}^{2}=K_{n}\left\|\sigma_{c, \mathbb{R}_{n}}\right\|_{R}^{2} .
\end{gathered}
$$

Hence, we conclude from above that

we have

$$
\lim _{n \rightarrow \infty}\left\|\sigma_{c, \bar{R}_{n}} \circ f_{n}-\sigma_{c, \bar{R}_{0}}\right\|_{R_{0}}=0 .
$$

Since

$$
\begin{gathered}
\sqrt{2}\left\|\sigma_{c, \overline{\mathrm{R}}_{n}} \circ f_{n}-\sigma_{c, \overline{\mathrm{R}}_{0}}\right\|_{R_{0}} \\
=\left\|\left[a_{n} \circ f_{n} \cdot\left(f_{n}\right)_{z}+\overline{a_{n}} \circ f_{n} \cdot \overline{\left(f_{n}\right)_{\bar{z}}}-a_{0}\right] d z\right\|_{R_{0}},
\end{gathered}
$$

$$
\begin{gathered}
\left\|\theta_{c, \bar{R}_{n}} \circ f_{n}-\theta_{c, \bar{R}_{0}}\right\|_{R_{0}} \\
\leqq\left\|\left[a_{n} \circ f_{n} \cdot\left(f_{n}\right)_{z}-a_{0}\right] d z\right\|_{R_{0}}+\left\|a_{n} \circ f_{n} \cdot\left(f_{n}\right)_{\bar{z}} d \bar{z}\right\|_{R_{0}} \\
\leqq \sqrt{2}\left\|\sigma_{c, \bar{R}_{n}} \circ f_{n}-\sigma_{c, \bar{R}_{0}}\right\|_{R_{0}}+2\left\|a_{n} \circ f_{n} \cdot\left(f_{n}\right)_{\bar{z}} d \bar{z}\right\|_{R_{0}} .
\end{gathered}
$$

Here $\left\|a_{n} \circ f_{n} \cdot\left(f_{n}\right)_{\bar{z}} d \bar{z}\right\|_{R_{0}}$ also converges to 0 , because

$$
\begin{aligned}
& \left\|a_{n} \circ f_{n} \cdot\left(f_{n}\right)_{\bar{z}} d \bar{z}\right\|_{R_{0}} \leqq k_{n}\left\|a_{n} \circ f_{n} \cdot\left(f_{n}\right)_{z} d z\right\|_{R_{0}} \\
\leqq & k_{n}\left\|\theta_{c, \bar{R}_{n}} \circ f_{n}\right\|_{R_{0}} \leqq \sqrt{2} k_{n}\left(\frac{1+k_{n}^{2}}{1-k_{n}^{2}}\right)^{1 / 2}\left\|\sigma_{c, \bar{R}_{n}}\right\|_{R_{n}},
\end{aligned}
$$

where $k_{n}=\sup _{R_{0}}\left|\left(f_{n}\right)_{\bar{z}}\right| /\left|\left(f_{n}\right)_{z}\right|$.

Thus if every $f_{n}$ is canonical, we have (6), and the assertion follows if we use Proposition 3. 


\section{A remark for the case of compact Riemann surfaces}

3.1. For a compact Riemann surface $R^{*}$ of genus $g(\geqq 2), T\left(R^{*}\right)$ is usually denoted by $T_{g}$, and we have the following corollary to Theorem 5 , which is essentially due to Ahlfors.

Corollary 6. Let $\bar{R}_{n} \in T_{g}$ converge to $\bar{R}_{0} \in T_{g}$, and a non-dividing simple closed curve $c$ be given. Then for every admissible sequence $\left\{f_{n}\right\}_{n=1}^{\infty}$ we have

$$
\lim _{n \rightarrow \infty}\left\|\theta_{c, \bar{R}_{n}} \circ f_{n}-\theta_{c, \bar{R}_{0}}\right\|_{R_{0}}=0 .
$$

If we let $G^{*}$ be a Fuchsian group corresponding to $R^{*}, T_{g}$ can be identified with the Teichmüller space of $G^{*}$, and is considered to be embedded in the (finite dimensional) space of $G^{*}$-invariant bounded holomorphic quadratic forms on the lower half plane (cf. [3], [7]). In the sequel we fix a non-dividing simple closed curve $c$ on $R^{*}$ (hence for every $\bar{R} \in T_{g}$ ), and by using the same notation and terminology as in [17], we shall consider the space $\partial_{c} T_{g}$ for $c$ and the fine topology on ${ }_{c} \hat{T}_{g}=$ $T_{g} \cup \partial_{c} T_{g}$. Here we recall some definitions. First, $\partial_{c} T_{g}$ is the set of marked Riemann surfaces with one single node corresponding to $c$. Next, by letting $S_{c}$ be the set of points $\bar{R}$ of $T_{g}$ on which $\theta_{c, \bar{R}}^{2}$ has a closed trajectory freely homotopic to $c$, we can construct a mapping $F_{1}$ from $S_{c}$ onto $T_{g-1,2}$, and set

$$
F_{2}(\bar{R})=\operatorname{Re} \int_{B_{1}} \frac{2 \cdot \theta_{c, \bar{R}}}{\left\|\theta_{c, \bar{R}}\right\|^{2}}+\sqrt{-1} \cdot m_{\bar{R}}
$$

for every $\bar{R} \in S_{c}$, where $m_{\bar{R}}$ is the modulus of the characteristic ring domain of $\theta_{c, \bar{R}}^{2}$ for $c$ on $R$ and $\left\{A_{j}, B_{j}\right\}_{j=1}^{g}$ is a canonical homology basis on $R$ such that $A_{1}$ is freely homotopic to $c$. Then $F=\left(F_{1}, F_{2}\right)$ can be extended to a bijection from $S_{c} \cup \partial_{c} T_{g}$ onto $T_{g-1} \times \hat{U}$, where $\hat{U}=\{z: \operatorname{Im} z>0\} \cup\{\infty\}$ is equipped with the usual fine (cusp) topology. The fine topology is, by definition, the induced topology by $F$ from $T_{g-1,2} \times \hat{U}$.

In this case we say that a sequence $\left\{\left\langle\bar{R}_{n}, \bar{R}_{0}, f_{n}\right\rangle_{n=1}^{\infty}\right.$ of deformations (cf. [1]) is admissible if for every neighbourhood $K$ of the (single) node of $R_{0}$ and every positive $\varepsilon$ there exists an $N$ such that $\left.f_{n}^{-1}\right|_{\left(R_{0}-K\right)}$ is $(1+\varepsilon)$-quasiconformal for every $n \geqq N$ (cf. Chapter 2), and set

$$
\begin{aligned}
& \theta_{\bar{R}}=\frac{2 \cdot \theta_{c, \bar{R}}}{\left\|\theta_{c, \bar{R}}\right\|_{R}^{2}} \text { for every } \quad \bar{R} \in T_{g}, \text { and } \\
& \theta_{\bar{R}}=\frac{\sqrt{-1}}{2 \pi} \cdot \varphi_{\bar{R}^{\prime}} \quad \text { for every } \quad \bar{R} \in \partial_{c} T_{g},
\end{aligned}
$$

where $\bar{R}^{\prime}=F_{1}(\bar{R})$ and $\varphi_{\bar{R}^{\prime}}$ is the elementary differential of the third kind on $\bar{R}^{\prime}$ with poles at two punctures. 
It is known that if $\bar{R}_{n} \in T_{g}$ converges to $\bar{R}_{0} \in \partial_{c} T_{g}$ in the sense of the conformal topology (cf. [1]), then it holds that

$$
\lim _{n \rightarrow \infty}\left\|\theta_{\bar{R}_{n}}\right\|_{R_{n}}=+\infty, \text { that is, } \lim _{n \rightarrow \infty}\left\|\theta_{c, \bar{R}_{n}}\right\|_{R_{n}}=0 .
$$

Now using the fine topology on ${ }_{c} \hat{T}_{g}$, we can extend this slightly by stating the main theorem of this section.

Theorem 7. Suppose that $\bar{R}_{n} \epsilon_{c} \hat{T}_{g}$ converges to $\bar{R}_{0} \epsilon_{c} \hat{T}_{g}$ in the sense of the fine topology. Then for every admissible sequence $\left\{\left\langle\bar{R}_{n}, \bar{R}_{0}, f_{n}\right\rangle\right\}_{n=1}$ of deformations we have

$$
\lim _{n \rightarrow \infty}\left\|\theta_{\bar{R}_{n}} \circ f_{n}^{-1}-\theta_{\bar{R}_{0}}\right\|_{\left(R_{0}-K\right)}=0
$$

for every neighbourhood $K$ of the node of $R_{0}$, where we assume that $K=\emptyset$ if $\bar{R}_{0} \in T_{g}$.

Note that (9) is derived from (10), because

$$
\begin{gathered}
\left\|\theta_{\bar{R}_{0}}\right\|_{\left(R_{0}-K\right)} \leqq\left\|\theta_{\bar{R}_{n}} \circ f_{n}^{-1}-\theta_{\bar{R}_{0}}\right\|_{\left(R_{0}-K\right)}+\left\|\theta_{\bar{R}_{n}} \circ f_{n}^{-1}\right\|_{\left(R_{0}-K\right)}, \\
\left\|\theta_{\bar{R}_{n}} \circ f_{n}^{-1}\right\|_{\left(R_{0}-K\right)}^{2} \leqq \frac{1+k_{n}^{2}}{1-k_{n}^{2}}\left\|\theta_{\bar{R}_{n}}\right\|_{\left(R_{n}-f_{n}^{-1}(K)\right)}^{2},
\end{gathered}
$$

and $\left\|\theta_{\bar{R}_{0}}\right\|_{\left(R_{0}-K\right)}$ tends to $+\infty$ as $K$ shrinks to the node of $R_{0}$.

3.2. The proof of Theorem 7 will be given in Section 3.3 after preparing the lemmas below. First we construct an admissible sequence satisfying the condition (10). Our construction of such a sequence is based on Corollary 6 and for later use we modify it as follows.

Lemma 3. Suppose that $\bar{R}_{n} \in T_{g}$ converges to $\bar{R}_{0} \in T_{g}$. Let $f_{\bar{R}_{n}}$ be the Teichmüller mapping of $\bar{R}_{0}$ to $\bar{R}_{n}$, and $f_{n}=f_{\bar{R}_{n}}^{-1}$. Then the admissible sequence $\left\{\left\langle\bar{R}_{n}, \bar{R}_{0}, f_{n}\right\rangle\right\}_{n=1}$ satisfies the condition (10).

Proof. It is clear that the given sequence is admissible, and noting that $\lim _{n \rightarrow \infty}\left\|\theta_{c, \bar{R}_{n}}\right\|_{R_{n}}=\left\|\theta_{c, \bar{R}_{0}}\right\|_{R_{0}}$ (cf. Corollary 4 ), the assertion follows from Corollary 6 and the following inequality:

$$
\begin{gathered}
\left\|\theta_{\bar{R}_{n}} \circ f_{n}^{-1}-\theta_{\bar{R}_{0}}\right\|_{R_{0}} \\
\leqq\left|\frac{2}{\left\|\theta_{c, \bar{R}_{n}}\right\|_{R_{n}}^{2}}-\frac{2}{\left\|\theta_{c, \bar{R}_{0}}\right\|_{R_{0}}^{2}}\right| \cdot\left\|\theta_{c, \bar{R}_{0}}\right\|_{R_{0}} \\
+\frac{2}{\left\|\theta_{c, \bar{R}_{n}}\right\|_{R_{n}}^{2}} \cdot\left\|\theta_{c, \bar{R}_{n}} \circ f_{n}^{-1}-\theta_{c, \bar{R}_{0}}\right\|_{R_{0}} .
\end{gathered}
$$

Now let a positive $y_{0}$ be fixed, and write

$$
X=\left\{\bar{R} \in S_{c}: F_{2}(\bar{R})=\sqrt{-1} \cdot y_{0}\right\} .
$$

Let $\left\{\bar{S}_{n}\right\} \subset X$ be a sequence converging to $\bar{S}_{0} \in X$, and $\bar{R}_{n}^{\prime}=F_{1}\left(\bar{S}_{n}\right)$ for every $n$. 
Then $\bar{R}_{n}^{\prime}$ is canonically embedded in a compact Riemann surface, say $\bar{R}_{n}$, of genus $g-1$, and $\bar{R}_{n}-R_{n}^{\prime}$ consists of two points, say $\left\{p_{n}^{i}\right\}_{i=1}^{2}$, for every $n$. Next let $\bar{R}_{n}=$ $F^{-1}\left(\bar{R}_{n}^{\prime}, \infty\right)$. Then $\theta_{\bar{R}_{n}}$ is, by definition, $(\sqrt{-1} / 2 \pi) \cdot \varphi_{\bar{R}_{n}^{\prime}}$. Denote the characteristic disk of $\theta_{\bar{R}_{n}}$ at $p_{n}^{i}$ on $\bar{R}_{n}$ by $D_{n}^{i}(i=1,2)$. Map each $D_{n}^{i}$ conformally onto $D=\{|z|<1\}$ so that $p_{n}^{i}$ corresponds to $z=0$. Then under the natural embedding $\pi_{n}$ of $S_{n}-C_{\bar{S}_{n}}$ into $R_{n}^{\prime}$ (where $C_{S_{n}}$ is the center trajectory of the characteristic ring domain $W_{S_{n}}$ of $\theta_{c, \bar{S}_{n}}$ for $c$ on $\bar{S}_{n}^{n}$ ), the images of the boundaries of $S_{n}-C_{\bar{S}_{n}}$ are loops in $D_{n}^{1}$ and $D_{n}^{2}$ corresponding to the circle $\left\{|z|=r_{0}\right\}$ in $D$ for every $n$ with $r_{0}=\exp \left[-\pi y_{0}\right]$. Finally let $W_{r, n}$ be the ring domain in $W_{S_{n}} \subset S_{n}$ containing $C_{S_{n}}$ such that the boundaries of $W_{r, n}$ are mapped by $\pi_{n}$ to the loops in $D_{n}^{1}$ and $D_{n}^{2}$ corresponding to $\{|z|=r\}$ in $D$, where $r_{0}<r<1$. (See [16], [17] for more detailed preliminaries.)

Lemma 4. Let $\bar{S}_{n}, \bar{R}_{0}$ and $W_{r, n}$ be as above, and let a finite number of constants $\left\{\delta_{j}\right\}_{j=1}^{s}$ such that $0<\delta_{1}<\delta_{2}<\ldots<\delta_{s}<\left(1-r_{0}\right)$ be given. Then there exists a sequence $\left\{\left\langle\bar{S}_{n}, \bar{R}_{0}, f_{n}\right\rangle\right\}_{n=1}^{\infty}$ of deformations satisfying the following condition: for every positive $\varepsilon\left(<1-r_{0}-\delta_{s}\right)$ we can find an $N$ such that for every $n \geqq N$

(a) $\left.f_{n}^{-1}\right|_{\left(R_{0}-K_{1}\right)}$ is $(1+\varepsilon)$-quasiconformal,

(b) $\left\|\theta_{\bar{S}_{n}} \circ f_{n}^{-1}-\theta_{\bar{R}_{0}}\right\|_{\left(R_{0}-K_{1}\right)}<\varepsilon$, and

(c) $f_{n}^{-1}\left(K_{j}\right) \supset W_{r_{j}, n}(j=1, \ldots, s)$ and $f_{n}^{-1}\left(N\left(R_{0}\right)\right)=C_{S_{n}}$,

where $K_{j}$ is the neighbourhood of the node $N\left(R_{0}\right)$ of $R_{0}$ corresponding to $\left\{|z|<r_{0}+\delta_{j}+\varepsilon\right\}$ in $D$, and $r_{j}=r_{0}+\delta_{j}$.

Proof. Let $f_{\bar{S}_{n}}$ be the Teichmüller mapping from $\bar{S}_{0}$ to $\bar{S}_{n}$. Then it is known ([16] Corollary 2) that we can find and $N_{1}$ such that

$$
f_{\bar{S}_{n}}\left(W_{r_{j}^{\prime}, 0}\right) \supset W_{r_{j}, n} \quad(j=1, \ldots, s)
$$

for every $n \geqq N_{1}$, where $r_{j}^{\prime}=r_{j}+\varepsilon$. Hence in particular we can consider each $\left.\pi_{0} \circ f_{S_{n}}^{-1}\right|_{\left(S_{n}-C_{S_{n}}\right)}\left(n \geqq N_{1}\right)$ as a quasiconformal embedding from $S_{n}-C_{S_{n}}$ into $R_{0}^{\prime} \subset R_{0}$, which we denote by $g_{n}$. Here we can assume (cf. [16]) that $\pi_{0}$ is conformally extended to a suitable neighbourhood of the boundaries of $S_{0}-C_{\bar{S}_{0}}$ in $W_{\bar{S}_{0}}$.

By deforming $g_{n}$ in $W_{r_{1}, n}-C_{\bar{S}_{n}}$, we can now make a deformation $\left\langle\bar{S}_{n}, \bar{R}_{0}, f_{n}\right\rangle$ such that $f_{n}^{-1}\left(N\left(R_{0}\right)\right)=C_{S_{n}}$ and $f_{n}$ coincides with $g_{n}$ on $S_{n}-W_{r_{1}, n}$ for every $n \geqq N_{1}$. By taking any suitable deformations for $n<N_{1}$, we arrive at a sequence of deformations. We show that this sequence satisfies the conditions in Lemma 4.

First by Lemma 3 for a given $\varepsilon$ we can find an $N\left(\geqq N_{\mathrm{i}}\right)$ such that

$$
f_{S_{n}} \text { is }(1+\varepsilon) \text {-quasiconformal, and }
$$

$$
\left\|\theta_{S_{n}} \circ f_{S_{n}}-\theta_{S_{0}}\right\|_{S_{0}}<\varepsilon \text { for every } n \geqq N \text {. }
$$

Note that $\pi_{0}\left(S_{0}-W_{r_{j}^{\prime}, n}\right)=R_{0}-K_{j}(j=1, \ldots, s)$ and $\theta_{\bar{S}_{0}} \circ \pi_{0}^{-1}=\theta_{\bar{R}_{0}}$ on $R_{0}-K_{1}$. Hence from (11) we have

$$
f_{n}\left(S_{n}-W_{r_{j}, n}\right)=g_{n}\left(S_{n}-W_{r_{j}, n}\right) \supset \pi_{0}\left(S_{0}-W_{r_{j}^{\prime}, 0}\right)=R_{0}-K_{j},
$$


that is, $f_{n}^{-1}\left(R_{0}-K_{j}\right) \subset S_{n}-W_{\boldsymbol{r}_{j}, n}(j=1, \ldots, s)$ for every $n \geqq N_{1}$. Thus we conclude that $f_{n}^{-1}\left(K_{j}\right) \supset W_{r_{j}, n}(j=1, \ldots, s)$ for every $n \geqq N_{1}$, which implies that $f_{n}^{-1}=$ $f_{\bar{S} n}^{-1} \circ \pi_{0}^{-1}$ on $R_{0}-K_{1}$, and hence $\left.f_{n}^{-1}\right|_{\left(R_{0}-K_{1}\right)}$ is $(1+\varepsilon)$-quasiconformal for every $n \geqq N$ by (12).

Next, from (13) we have

$$
\begin{gathered}
\left\|\theta_{\bar{S}_{n}} \circ f_{n}^{-1}-\theta_{\bar{R}_{0}}\right\|_{\left(R_{0}-K_{1}\right)}=\left\|\theta_{\bar{S}_{n}} \circ f_{\bar{S}_{n}} \circ \pi_{0}^{-1}-\theta_{\bar{S}_{0}} \circ \pi_{0}^{-1}\right\|_{\left(R_{0}-K_{1}\right)} \\
=\left\|\theta_{\bar{S}_{n}} \circ f_{\bar{S}_{n}}-\theta_{\bar{S}_{0}}\right\|_{\left(S_{0}-W r_{1}^{\prime}, 0\right)}<\varepsilon
\end{gathered}
$$

for every $n \geqq N$, which completes the proof.

Now we can show the following

Lemma 5. Suppose that $\bar{R}_{n} \in \partial_{c} T_{g}$ and converges to $\bar{R}_{0} \in \partial_{c} T_{g}$. Then there exists an admissible sequence $\left\{\left\langle\bar{R}_{n}, \bar{R}_{0}, f_{0}\right\rangle\right\}_{n=1}^{\infty}$ of deformations satisfying the condition (10).

Proof. Let $K_{n}^{k}$ be the neighbourhood of the node of $R_{n}$ corresponding to $\{|z|<1 / k\}$ in $D$ for every $n$. Fix a positive integer $k$ arbitrarily, and let $\bar{R}_{k, n}=$ $F^{-1}\left(\bar{R}_{n}^{\prime},(\sqrt{-1} / \pi) \log (k+1)\right)$, where $\bar{R}_{n}^{\prime}=F_{1}\left(\bar{R}_{n}^{\prime}\right)$. Then using Lemma 4 with $\bar{S}_{n}=\bar{R}_{k, n}, y_{0}=(1 / \pi) \log (k+1) \quad\left(\right.$ hence $\left.r_{0}=1 /(k+1)\right), \quad s=1$, and $\delta_{1}=\varepsilon=\varepsilon_{k}=$ $(1 / 2)(1 / k-1 /(k+1))$, we have a sequence $\left\{\left\langle\bar{R}_{k, n}, \bar{R}_{0}, f_{k, n}\right\rangle\right\}_{n=1}^{\infty}$ of deformations satisfying the condition; there exists an $N_{k}$ such that for every $n \geqq N_{k}$

(i) $\left.f_{k, n}^{-1}\right|_{\left(R_{0}-K_{0}^{k}\right)}$ is $\left(1+\varepsilon_{k}\right)$-quasiconformal,

(ii) $\left\|\theta_{\bar{R}_{k}, n} \circ f_{k, n}^{-1}-\theta_{\bar{R}_{0}}\right\|_{\left(R_{0}-K_{0}^{k}\right)}^{k}<\varepsilon_{k}$, and

(iii) $f_{k, n}^{-1}\left(K_{0}^{k}\right) \supset W_{r_{1}, n} \quad\left(\right.$ with $\left.\quad r_{1}=\frac{1}{2}\left(\frac{1}{k}+\frac{1}{k+1}\right)\right)$ and $f_{k, n}^{-1}\left(N\left(R_{0}\right)\right)=C_{\bar{R}_{k}, n}$.

Now let $\pi_{k, n}$ be the natural embedding of $R_{k, n}-C_{\bar{R}_{k, n}}$. Then we have an embedding $f_{k, n} \circ \pi_{k, n}^{-1}$ from $R_{n}-K_{n}^{k+1}$ into $R_{0}$ for every $n \geqq N_{k}$, and deforming $\pi_{k, n}$ in $W_{r_{1}, n}-C_{\bar{R}_{k, n}}$ we can make a sequence $\left\{\left\langle\bar{R}_{n}, \bar{R}_{0}, g_{k, n}\right\rangle\right\}_{n=N_{k}}^{\infty}$ of deformations such that $g_{k, n}=f_{k, n} \circ \pi_{k, n}^{-1}$ on $\pi_{k, n}\left(R_{k, n}-W_{r_{1}, n}\right)$, which contains $g_{k, n}^{-1}\left(R_{0}-K_{0}^{k}\right)$ by (iii). As in the proof of Lemma 4 , it is easily seen from (i) and (ii) that

(iv) $\left.g_{k, n}^{-1}\right|_{\left(R_{0}-K_{0}\right)}$ is $\left(1+\varepsilon_{k}\right)$-quasiconformal, and

(v) $\left\|\theta_{\bar{R}_{n}} \circ g_{k, n}^{-1}-\theta_{\bar{R}_{0}}\right\|_{\left(R_{0}-K_{0}^{k}\right)}<\varepsilon_{k}$

for every $n \geqq N_{k}$. Here we assume that $N_{k+1} \geqq N_{k}$ for every $k$.

Finally define a sequence $\left\{\left\langle\bar{R}_{n}, \bar{R}_{0}, f_{n}\right\rangle\right\}_{n=1}^{\infty}$ of deformations by taking as $f_{n}$ the above $g_{k, n}$ if $N_{k} \leqq n \leqq N_{k+1}-1$. By (iv) and (v) we find that it is a desired sequence.

Lemma 6. Suppose that $\bar{S}_{n} \in T_{g}$ and converges to $\bar{R}_{0} \in \partial_{c} T_{g}$. Then there exists an admissible sequence $\left\{\left\langle\bar{S}_{n}, \bar{R}_{0}, f_{n}\right\rangle\right\}_{n=1}^{\infty}$ of deformations satisfying the condition (10). 
Proof. From the definition of the fine topology ([17]), we may assume that $\left\{\bar{S}_{n}\right\}_{n=1}^{\infty}$ is contained in $S_{c}$. Let $F\left(\bar{S}_{n}\right)=\left(\bar{R}_{n}^{\prime}, z_{n}\right)$ and $\bar{R}_{n}=F^{-1}\left(\bar{R}_{n}^{\prime}, \infty\right)$ for every $n$, and $K_{n}^{k}$ and $\varepsilon_{k}$ be as in the proof of Lemma 5. Then since $\bar{R}_{n}$ converges to $\bar{R}_{0}$, using Lemma 4 as in the proof of Lemma 5 , we can construct a sequence $\left\{\left\langle\bar{R}_{n}, \bar{R}_{0}, g_{n}\right\rangle_{\}_{n=1}}^{\infty}\right.$ of deformations satisfying the condition: for every positive integer $k$, there exists an $N_{k}$ such that for every $n \geqq N_{k}$

(i) $\left.g_{n}^{-1}\right|_{\left(R_{0}-K_{0}^{k}\right)}$ is $\left(1+\varepsilon_{k}\right)$-quasiconformal,

(ii) $\left\|\theta_{\bar{R} n} \circ g_{n}^{-1}-\theta_{\bar{R}_{0}}\right\|_{\left(R_{0}-K_{0}^{k}\right)}<\varepsilon_{k}$, and

(iii) $g_{n}^{-1}\left(K_{0}^{j}\right) \supset K_{n}^{j+1} \quad(j=1, \ldots, k)$.

Next, let $k_{n}$ be the largest integer such that $\pi_{n}\left(S_{n}-C_{\bar{S}_{n}}\right) \cap K_{n}^{k} \neq \emptyset$ (that is, $\left.\pi_{n}\left(S_{n}-C_{\bar{S}_{n}}\right) \supset R_{n}-K_{n}^{k}\right)$ for every $n$. Then $k_{n}$ tends to $+\infty$, for $1 / k_{n}>\exp \left(-\pi \cdot \operatorname{Im} z_{n}\right)$ for every $n$ and $\lim _{n \rightarrow \infty} \operatorname{Im} z_{n}=+\infty$. Deform each $\pi_{n}$ in $\pi_{n}^{-1}\left(K_{n}^{k_{n}}\right)$ as before, and we have a sequence $\left\{\left\langle\bar{S}_{n}, \bar{R}_{n}, h_{n}\right\rangle\right\}_{n=1}^{\infty}$ of deformations such that $h_{n}\left(=\pi_{n}\right)$ is conformal on $S_{n}-\pi_{n}^{-1}\left(K_{n}^{k_{n}}\right)$.

Finally let $f_{n}=g_{n} \circ h_{n}$ for every $n$. We can now show that $\left\{\left\langle\bar{S}_{n}, \bar{R}_{0}, f_{n}\right\rangle\right\}_{n=1}^{\infty}$ is a desired sequence. In fact, for every neighbourhood $K$ of the node of $R_{0}$ and every positive $\varepsilon$, there exists a $k_{0}$ such that $K \supset K_{0}^{k_{0}}$ and $\varepsilon_{k_{0}}<\varepsilon$. Thus we can find an $N\left(\geqq N_{k_{0}}\right.$ ) such that $k_{n} \geqq k_{0}+1$ (, hence $f_{n}^{-1}(K) \supset h_{n}^{-1}\left(K_{n}^{k_{n}}\right)$ by (iii)), and (i) and (ii) hold with $k=k_{0}$ for every $n \geqq N$. Then noting that $f_{n}^{-1}=\pi_{n}^{-1} \circ g_{n}^{-1}$ and $\theta_{\bar{S}_{n}} \circ \pi_{n}^{-1}=\theta_{\bar{R}_{n}}$ on $S_{n}-f_{n}^{-1}(K)$ for every $n \geqq N$, we have the assertion.

3.3. Proof of Theorem 7. We consider only the case in Lemma 6, for other cases can be treated similarly as in Proposition 3. Let $\bar{S}_{n}, \bar{R}_{0}$ and $f_{n}$ be as in Lemma 6, $G_{n} \in T\left(G^{*}\right)$ correspond to $\bar{S}_{n}$, and let $U_{n}$ be the component of $G_{n}$ such that $\bar{S}_{n}=U_{n} / G_{n}$. Also let $G_{0} \in \partial T\left(G^{*}\right)$ correspond to $\bar{R}_{0}, U_{0}$ be a non-invariant component of $G_{0}$, and $F_{n}$ be the lift of $f_{n}^{-1}$ from $U_{0}$ into $U_{n}$. Then it is seen (cf. [1]) that $F_{n}$ converges locally uniformly on $U_{0}$ to the identity, and $U_{0}$ is a component of the Carathéodory kernel of $\left\{U_{n}\right\}_{n=1}^{\infty}$.

Let $a_{n}(z) d z$ and $a_{0}(z) d z$ be the lifts of $\theta_{\bar{S}_{n}}$ on $U_{n}$ and $\theta_{\bar{R}_{0}}$ on $U_{0}$, respectively, and fix $z_{0} \in U_{0}$ and a small $\varrho$ such that $D_{2 \varrho}=\left\{\left|z-z_{0}\right| \leqq 2 \varrho\right\}$ is contained in $U_{0}$ (hence, in $U_{n}$ for every sufficiently large $n$ ) arbitrarily. Then similarly as in the proof of Lemma 2 in [2], we can show that there exists an $M$ such that $\sup _{z \in D_{2 \varrho}}\left|a_{n}(z)\right| \leqq M$ for every sufficiently large $n$. It also holds that

$$
\begin{gathered}
\left|a_{n}\left(z_{0}\right)-a_{0}\left(z_{0}\right)\right| \leqq \frac{1}{\sqrt{2 \pi \varrho^{2}}}\left\|a_{n}(z)-a_{0}(z)\right\|_{2, D_{e}} \\
\leqq \frac{1}{\sqrt{2 \pi \varrho^{2}}}\left[\left\|a_{n}(z)-a_{n}\left(F_{n}(z)\right)\right\|_{2, D_{e}}+\left\|a_{n}\left(F_{n}(z)\right)\left[\left(F_{n}\right)_{z}-1\right]\right\|_{2, D_{e}}\right. \\
\left.+\left\|a_{n}\left(F_{n}(z)\right)\left(F_{n}\right)_{z}-a_{0}(z)\right\|_{2, D_{e}}\right] .
\end{gathered}
$$


Hence using Lemma 6 and Theorem V 5-3 in [14], we can show that $a_{n}(z)$ converges to $a_{0}(z)$ pointwise, hence locally uniformly on $U_{0}$.

Finally take any admissible sequence $\left\{\left\langle\bar{S}_{n}, \bar{R}_{0}, f_{n}^{\prime}\right\rangle\right\}_{n=1}^{\infty}$ and neighbourhood $K$ of the node of $R_{0}$, and fix them. Let $F_{n}^{\prime}$ be the lift of $f_{n}^{\prime-1}$ from $U_{0}$ into $U_{n}$, and $E$ be a relatively compact region in $U_{0}$ which covers $R_{0}-K$. It is then easily seen from above that $a_{n}\left(F_{n}^{\prime}(z)\right)$ is uniformly bounded on $E$ and $a_{n}\left(F_{n}^{\prime}(z)\right)$ converges to $a_{0}(z)$ uniformly on $E$. Hence from the inequality

$$
\begin{gathered}
\left\|\theta_{\bar{S}_{n}} \circ f_{n}^{\prime-1}-\theta_{\bar{R}_{0}}\right\|_{\left(R_{0}-K\right)} \leqq\left\|a_{n}\left(F_{n}^{\prime}(z)\right)\left[\left(F_{n}^{\prime}\right)_{z}-1\right]\right\|_{2, E} \\
+\left\|a_{n}\left(F_{n}^{\prime}(z)\right)-a_{0}(z)\right\|_{2, E}+\left\|a_{n}\left(F_{n}^{\prime}(z)\right)\left(F_{n}^{\prime}\right)_{\bar{z}}\right\|_{2, E}
\end{gathered}
$$

and Theorem V 5-3 in [14], we can conclude that

$$
\lim _{n \rightarrow \infty}\left\|\theta_{\bar{S}_{n}} \circ f_{n}^{\prime-1}-\theta_{\bar{R}_{0}}\right\|_{\left(R_{0}-K\right)}=0 .
$$

\section{References}

[1] AвIKoff, W.: Degenerating families of Riemann surfaces. - Ann. of Math. 105, 1977, 29-44.

[2] AhLfors, L. V.: The complex analytic structure of the space of closed Riemann surfaces. Analytic functions, edited by R. Nevanlinna, H. Behnke, H. Grauert, L. V. Ahlfors, D. C. Spencer, L. Bers, K. Kodaira, M. Heins and J. A. Jenkins, Princeton. New Jersey, Princeton University Press, 1960, 45-66.

[3] Ahlfors, L. V.: Lectures on quasiconformal mappings. - D. Van Nostrand Company, Inc., Princeton, New Jersey-Toronto-New York-London, 1966.

[4] Ahlfors, L. V., and L. SARIo.: Riemann surfaces. - Princeton, New Jersey, Princeton University Press, 1960.

[5] Ahlfors, L., and G. Weill: A uniqueness theorem for Beltrami equations. - Proc. Amer. Math. Soc. 13, 1962, 975-978.

[6] Bers, L.: Riemann surfaces. - Lecture Notes Published by the Courant Institute of Mathematical Sciences, New York University, 1957-58.

[7] Bers, L.: On boundaries of Teichmüller spaces and on kleinian groups: I. - Ann. of Math. $91,1970,570-600$.

[8] Earle, C.: Reduced Teichmüller spaces. - Trans. Amer. Math. Soc. 126, 1967, 54-63.

[9] KÜHNAU, R.: Verzerrungssätze und Koeffizientenbedingungen vom Grunskyschen Typ für quasikonforme Abbildungen. - Math. Nachr. 48, 1971, 77-105.

[10] Kusunoki, Y.: On Riemann's period relations on open Riemann sufaces. - Mem. Coll. Sci. Univ. Kyoto. Ser. A. Math. 30, 1956, 1-22.

[11] KusUnokI, Y.: Square integrable normal differentials on Riemann surfaces. - J. Math. Kyoto Univ. 3, 1963, 59-69.

[12] KUSUNOKI, Y.: Riemann surfaces and conformal mappings. - Asakura. Tokyo, 1973 (Japanese).

[13] Kusunoki, Y., and M. TANiguchi: Remarks on Fuchsian groups associated with open Riemann surfaces. - To appear in Ann. Math. Studies 97.

[14] Lehto, O., and K. I. VIRTANen: Quasikonforme Abbildungen. - Springer-Verlag BerlinHeidelberg-New York, 1965. 
[15] Lehto, O.: Schlicht functions with a quasiconformal extension. - Ann. Acad. Sci. Fenn. Ser. A I 500, 1971, 1-10.

[16] Taniguchi, M.: Abelian differentials whose squares have closed trajectories on compact Riemann surfaces. - Japan. J. Math. 4, 1978, 417-443.

[17] TANiguchI, M.: Remarks on topologies associated with squeezing a non-dividing loop on compact Riemann surfaces. - J. Math. Kyoto Univ. 19, 1979, 203-214.

\author{
Kyoto University \\ Faculty of Science \\ Department of Mathematics \\ Kyoto, 606 \\ Japan
}

Received 13 June 1979 